K.A. van der Hucht, G. Koenigsberger \& P.R.J. Eenens, eds.

\title{
Infrared observations of young massive stars
}

\author{
Bringfried Stecklum ${ }^{1}$, Markus Feldt ${ }^{2}$, Thomas Henning ${ }^{2}$, \\ and Werner Pfau ${ }^{2}$ \\ ${ }^{1}$ Thüringer Landessternwarte, \\ Sternwarte 5, D-07778 Tautenburg, Deutschland \\ ${ }^{2}$ Universitäts-Sternwarte $\&$ Astrophysikalisches Institut, \\ Schillergäßchen 2-3, D-07745 Jena, Deutschland
}

\begin{abstract}
Infrared observations of young massive stars yield crucial insights on the birth of high-mass stars and their interaction with the parent molecular cloud. Results for IRAS23140+6121, G254.681+0.219, and NGC6334F obtained by near- and mid-infrared imaging are presented for a brief illustration.
\end{abstract}

\section{Discussion}

The formation of high-mass stars $\left(M \geq 8 \mathrm{M}_{\odot}\right)$ is still a matter of debate. The validity of the accretion scenario has been questioned since radiation pressure on dust grains might hinder the infall of matter. The coalescence of medium-mass stars was suggested to be an alternative mechanism (Bonnell et al. 1998). Due to the large average distances of high-mass stars, ultimate angular resolution, e.g., by adaptive optics $(\mathrm{AO})$, is required for a detailed study of their formation. Their rapid evolution implies that they burn hydrogen while still being deeply embedded in the parent molecular cloud, giving rise to ultracompact HII
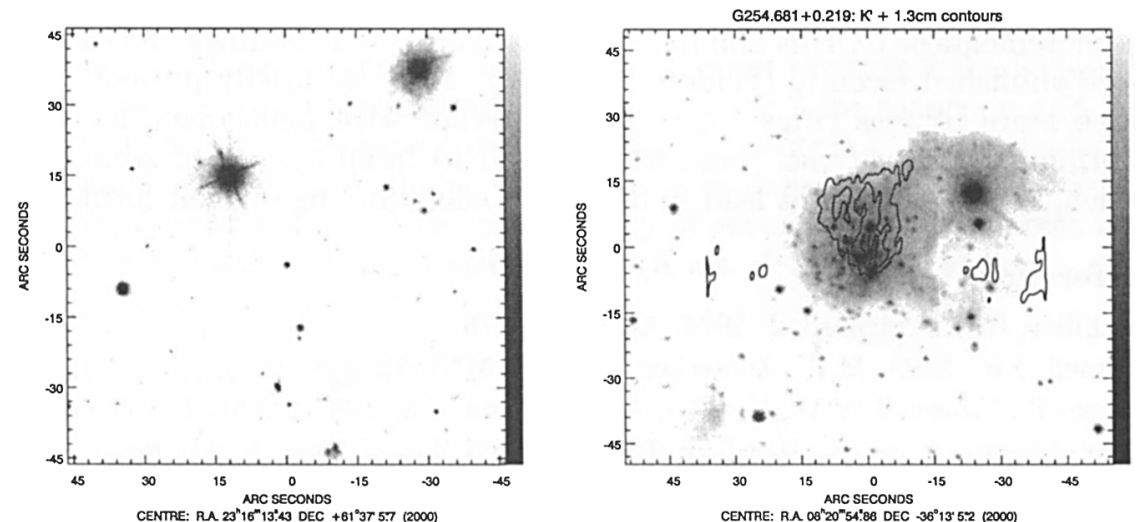

Figure 1. Left: AO-image of IRAS $23140+6121$ taken with ALFA at $2.1 \mu \mathrm{m}$. The wavefront reference star is at the position $\left(+12^{\prime \prime},+15^{\prime \prime}\right)$. The UCHII at ($\left.27^{\prime \prime},+42^{\prime \prime}\right)$ is marginally resolved. The polarisation of its diffuse emission points to scattering in bipolar cavities. The faint bipolar object at $\left(-6^{\prime \prime},-45^{\prime \prime}\right)$ coincides with intense $1.3 \mathrm{~mm}$ dust continuum emission (R. Klein, private comm.). Right: $2.2 \mu \mathrm{m}$ image of the UCHII G 254.681+0.219 with contours of the $1.3 \mathrm{~cm}$ emission from Kurtz (1995). The dense ionized gas is confined to the embedded star cluster. 

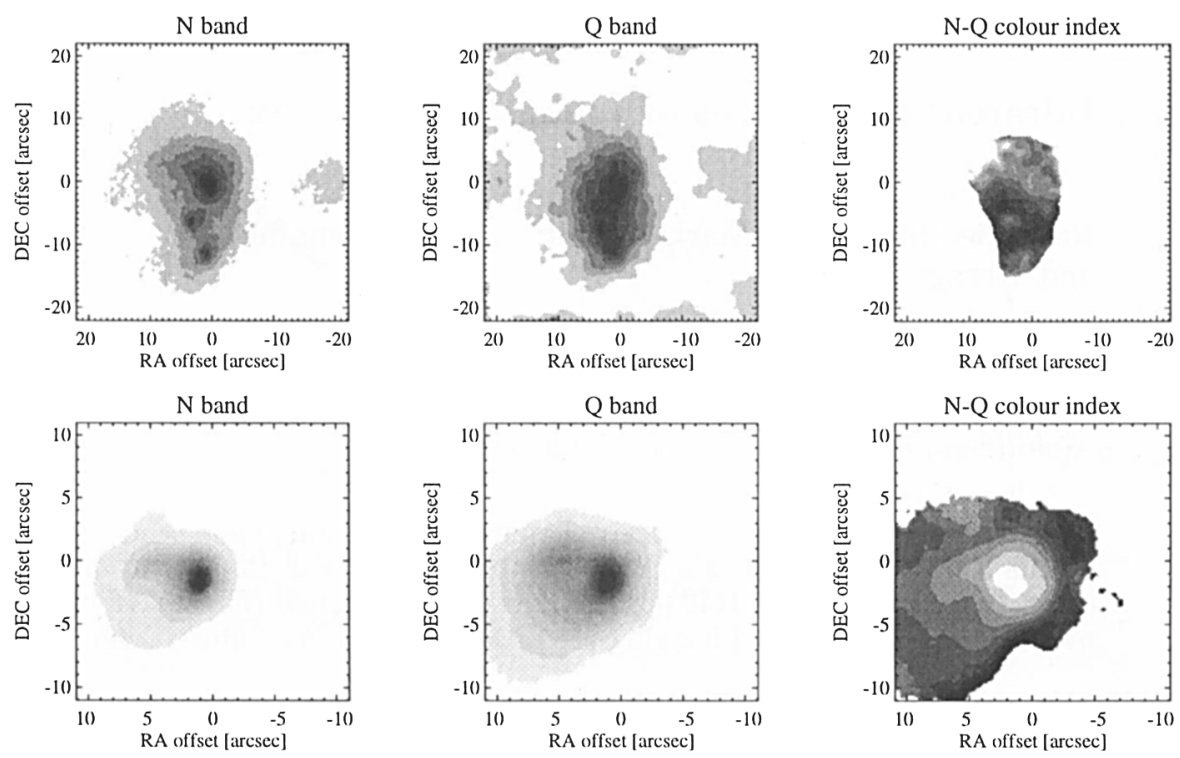

Figure 2. $N$ and $Q$ images of G 254.681+0.219 (top) and NGC 6334F (bottom) taken with MANIAC (Böker et al. 1997). The distribution of the thermal radiation from hot dust is similar to that of the free-free emission. Three heating sources are present in G 254.681+0.219. The right images show the $N-Q$ colour index where areas of low colour temperature appear dark. The deeply embedded outflowdriving source NGC 6334F-IRS2 (Bachiller \& Cernicharo 1990) is visible in the $Q$ image at $\left(-3^{\prime \prime}, 2^{\prime \prime}\right)$ and the $N-Q$ map. It was not yet detected at $\lambda \leq 10 \mu \mathrm{m}$ (Persi et al. 1998).

regions (UCHIIs). The large visual extinction towards such objects precludes their detection at optical wavelengths. We performed high-resolution infrared measurements of UCHIIs and hot molecular cores. Our findings on G 45.45+0.06 were published recently (Feldt et al. 1998). Here we briefly present results on three more sources (Figs. $1 \& 2)^{1}$. Together with molecular line and radio continuum observations, these will be used to build consistent source models, which, eventually, might lead to a better understanding of their formation.

\section{References}

Bachiller, R., Cernicharo, J. 1990, A\&A 239, 276

Bonnell, I.A., Bate, M.R., Zinnecker, H. 1998, MNRAS 298, 93

Böker, T., Storey, J.W.V., Krabbe, A., Lehmann, Th. 1997, PASP 109, 827

Feldt, M., Stecklum, B., Henning, Th., Hayward, T.L., Klein, R., Lehmann, Th. 1998, A\&A 339,759

Kurtz, S. 1995, RevMexAA-SC 3, 39

Molinari, S., Brand, J., Cesaroni, R., Palla, F., Palumbo, G. 1998, A\&A 336, 339

Persi, P., Tapia, M., Felli, M., Lagage, P., Ferrari-Toniolo, M. 1998, A\&A 336, 1024

\footnotetext{
${ }^{1}$ Based on observations collected at the European Southern Observatory, La Silla, Chile, and the German Spanish Astronomical Centre, Calar Alto, Spain.
} 